\title{
reciamuc
}

Revista científica de investigación actualización del mundo de las ciencias

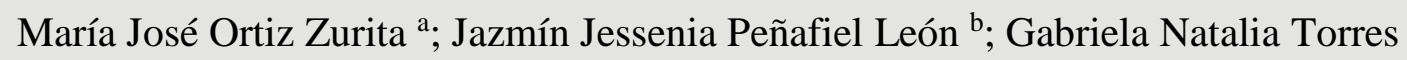
Jara $^{\text {c; }}$ Diana Valeria Delgado Campuzano ${ }^{\text {d; Evelyn Mariuxi Bautista Guaranda }}{ }^{\text {e }}$

Calidad educativa, entorno familiar y su relación con el rendimiento académico de la enseñanza secundaria en centros educativos de la provincia de Manabí durante año $2017-2018$

Educational quality, family environment and its relationship with the academic performance of secondary education in schools in the province of Manabi during 2017-2018

Revista Científica de Investigación actualización del mundo de las Ciencias. Vol. 3 núm., 2, abril, ISSN: 2588-0748, 2018, pp. 116-142

DOI: $10.26820 / \mathrm{reciamuc/3.(2).abril.2019.116-142}$

URL: http://reciamuc.com/index.php/RECIAMUC/article/view/357

Código UNESCO: 5802 Organización y Planificación de la Educación

Tipo de Investigación: Artículo de Investigación

Editorial Saberes del Conocimiento

Recibido: 21/02/2019 Aceptado: 10/03/2019 Publicado: 30/04/2019

Correspondencia: $\underline{\text { mortizz1@unemi.edu.ec }}$

a. Magister en Administración de Empresas; Master Universitario en Dirección y Planificación de Turismo; Ingeniera en Administración de Empresas Turísticas y Hoteleras; Docente Titular Universidad Estatal de Milagro; mortizz1@unemi.edu.ec

b. Master en Gestión Turística; Ingeniera en Administración de Empresas Turísticas y Hoteleras; Docente Titular Universidad Estatal de Milagro; jpenafiell@unemi.edu.ec

c. Master Universitario en Gestión del Patrimonio Cultural y Museología; Ingeniera en Gestión Turística y Hotelera; Docente Titular Universidad Estatal de Milagro; gtorresj@unemi.edu.ec

d. Magister en Administración Ambiental; Ingeniera en Ecoturismo; Docente Titular Universidad Estatal de Milagro; ddelgadoc@unemi.edu.ec

e. Licenciada en Turismo; evymar92@gmail.com 


\section{Calidad educativa, entorno familiar y su relación con el rendimiento académico de la enseñanza secundaria en centros educativos de la provincia de Manabí durante año 2017 -2018 \\ Vol. 3, núm. 2., (2019) \\ María José Ortiz Zurita; Jazmín Jessenia Peñafiel León; Gabriela Natalia Torres Jara; Diana \\ Valeria Delgado Campuzano; Evelyn Mariuxi Bautista Guaranda}

\section{RESUMEN}

Las sociedades actuales requieren de la educación integral de sus ciudadanos, con énfasis en el vínculo familia- escuela; de ahí la importancia de fomentar estudios que aporten a la comprensión de este fenómeno. La presente investigación comprueba la relación existente entre las variables: calidad educativa, entorno familiar y rendimiento académico. La misma se realizó en cinco escuelas secundarias de la provincia de Manabí. La muestra estuvo compuesta por 118 estudiantes, del tercer año de Bachillerato. Se empleó la metodología cuantitativa y un estudio descriptivo correlacional, donde se utilizó un instrumento valorativo- la encuesta-. Entre los resultados más relevantes se obtuvo: Existe una estrecha relación entre las variables calidad educativa y el entorno familiar, las características de ambos elementos son determinantes en el rendimiento académico de los estudiantes, según la percepción de los estudiantes. En este estudio, los sujetos de familia nucleares y que asisten a escuelas privadas y fisco-misionales obtuvieron un rendimiento académico más alto. Finalmente se propone un Plan de Mejoras para fortalecer las funciones de la escuela y la familia en aras de lograr un mejor rendimiento educativo.

Palabras Claves: Rendimiento académico; entorno familiar; calidad educativa. 


\section{Calidad educativa, entorno familiar y su relación con el rendimiento académico de la enseñanza secundaria en centros educativos de la provincia de Manabí durante año 2017 -2018}

Vol. 3, núm. 2., (2019)

María José Ortiz Zurita; Jazmín Jessenia Peñafiel León; Gabriela Natalia Torres Jara; Diana Valeria Delgado Campuzano; Evelyn Mariuxi Bautista Guaranda

\section{ABSTRACT}

Current societies require the integral education of their citizens, with emphasis on the familyschool link; hence the importance of encouraging studies that contribute to the understanding of this phenomenon. The present investigation verifies the relationship between the variables: educational quality, family environment and academic performance. It was carried out in five secondary schools in the province of Manabí. The sample consisted of 118 students from the third year of Bachillerato. The quantitative methodology and a correlational descriptive study were used, where a valorative instrument was used - the survey. Among the most relevant results was obtained: There is a close relationship between the educational quality variables and the family environment. The characteristics of both elements are determining factors in the academic performance of the students, according to the perception that students have. In the present study, the subjects belonging to nuclear families and who attend private and fiscal-missionaries schools obtained a higher academic performance. Finally, an Improvement Plan is proposed to strengthen the functions of the school and the family in order to achieve a better educational performance.

Key Words: Academic performance; family environment; educational quality. 


\section{Calidad educativa, entorno familiar y su relación con el rendimiento académico de la enseñanza secundaria en centros educativos de la provincia de Manabí durante año 2017 -2018 \\ Vol. 3, núm. 2., (2019) \\ María José Ortiz Zurita; Jazmín Jessenia Peñafiel León; Gabriela Natalia Torres Jara; Diana Valeria Delgado Campuzano; Evelyn Mariuxi Bautista Guaranda}

\section{Introducción.}

Históricamente una de las grandes preocupaciones que ha tenido el profesorado y la familia han sido las relaciones vinculares y las pautas de educación para la crianza de los hijos. Las últimas décadas han sido muy productivas en la conceptualización de los elementos didácticos y metodológicos presentes en el Proceso de Enseñanza Aprendizaje y de las relaciones que se establecen al interno de las instituciones educativas y el resto de los agentes socializadores. Siendo este tema objeto de investigación por parte de estudiosos de todo el mundo, sobresalen por sus aportes: González Rey (1998), Febles (2001), Domínguez (2005), Hernández (2015), Londoño (2017) y otros. Los temas más abordados en estos estudios son: relación y funciones de la escuela y de la familia. Merino y Morales (2002) también proponen niveles de participación de la familia en la escuela: 1) obligaciones básicas de la familia o parentalidad. Los padres son los encargados de la salud, bienestar familiar y seguridad del niño aquí entran principalmente las necesidades básicas. 2) obligaciones básicas de la escuela o comunicación, la relación y comunicación de padres de familia- maestros, donde los profesores deben de comentar con los progenitores cualquier tema relacionado con su hijo, en especial su nivel de aprendizaje, así como aspectos positivos y negativos que el niño tenga dentro y fuera del salón. 3) participación en la escuela o voluntariado, la escuela brinda a los padres de familia acceso a establecimientos de la institución para ayudar en actividades de mejoramiento; 4) involucrarse en actividades de aprendizaje en la casa, los profesores orientan a los padres de familia como ayudar a los alumnos en el hogar, en sus tareas y trabajos extra escolares; y los padres se responsabilizan de ser ellos quienes ayudaran al alumno para mejorar el rendimiento académico; 5) participación en la toma de decisiones de la institución. En nuestro país las políticas públicas apoyan el desarrollo de la educación ejemplo: Plan Nacional para el Buen Vivir 20142021, Plan Nacional de Desarrollo y el Plan para la erradicación de la pobreza. Políticas que apuntan a la formación integral de la ciudadanía.

El presente estudio contribuye con estas políticas al profundizar en el vínculo hogar-escuelarendimiento académico. Dota a los investigadores de herramientas teóricas- metodológicas y prácticas, fomentando habilidades científicas como: análisis crítico reflexivo, pensamiento holístico- desarrollador, metacognición adecuada, conciencia crítica orientada a la solución de problemas.

Los objetivos planteados en la investigación se cumplen pues permitió el análisis de una relación fundamental para el desarrollo de los adolescentes, el binomio escuela-familia. Realizar la presente 


\section{Calidad educativa, entorno familiar y su relación con el rendimiento académico de la enseñanza secundaria en centros educativos de la provincia de Manabí durante año 2017 -2018}

Vol. 3, núm. 2., (2019)

María José Ortiz Zurita; Jazmín Jessenia Peñafiel León; Gabriela Natalia Torres Jara; Diana Valeria Delgado Campuzano; Evelyn Mariuxi Bautista Guaranda

investigación contribuyó a la comprensión de los procesos docentes educativos sus fortalezas y debilidades. No obstante, enfrentamos varias limitaciones como son: El escaso tiempo con que se contó para hacer la investigación. La resistencia grupal, ofrecida por los participantes por el temor a ser cuestionados. La negación de la familia a aceptar que son responsables directos en el rendimiento académico de sus hijos.

Por ello, en esta investigación se profundiza en la relación entre la calidad educativa, el entorno familiar y el rendimiento académico, con una muestra compuesta por educandos en cinco instituciones educativas del nivel secundario de la provincia de Manabí, ubicada en la ciudad de Manta. El estudio es importante porque permitió constatar el estado actual de las instituciones de la enseñanza secundaria en aspectos como el estado de las instalaciones físicas, desempeño docente y gestión pedagógica, así como de los entornos familiares. Por lo cual se empleó la Metodología Cuantitativa. Específicamente un estudio descriptivo correlacional cuyos métodos utilizados fueron: estadístico, analítico-sintético, bibliográfico, hermenéutico, con el apoyo de las técnicas de la observación y la encuesta.

La presente investigación se divide en 3 capítulos: capítulo I se abordan los fundamentos teóricos que sirven de sustento al estudio, se definen conceptualmente las variables: calidad educativa, rendimiento académico, estándares de la calidad educativa, y entorno familiar, el capítulo II se declara el sustento metodológico de la investigación. Se determinan los métodos, técnicas e instrumentos a emplear, así como los recursos utilizados. Se definen los criterios de inclusión y la caracterización de la muestra, capítulo III: se realiza el análisis e interpretación de los resultados por ítems, a partir del uso del paquete estadístico SPSS y Excel. Se ofrece un análisis descriptivo y gráfico de cada una de las variables. Se proponen las conclusiones a las cuales se arriban luego de finalizada la investigación y las recomendaciones donde se indican los aspectos que deben ser fortalecidos por las instituciones de la enseñanza secundaria a fin de que brinden una educación de calidad.

\section{Metodología.}

La investigación se realizó en la provincia de Manabí, específicamente en el Cantón Manta. La matrícula del tercer año de bachillerato de las instituciones en estudio es de 400 estudiantes, la muestra quedó constituida finalmente por 118 estudiantes del tercer año de bachillerato, los mismos provienen de 


\section{Calidad educativa, entorno familiar y su relación con el rendimiento académico de la enseñanza secundaria en centros educativos de la provincia de Manabí durante año 2017 -2018}

Vol. 3, núm. 2., (2019)

María José Ortiz Zurita; Jazmín Jessenia Peñafiel León; Gabriela Natalia Torres Jara; Diana Valeria Delgado Campuzano; Evelyn Mariuxi Bautista Guaranda

las 5 instituciones colaboradoras en el estudio (de las cuales no se revela el nombre por políticas de privacidad de las escuelas).

Diseño de investigación

La investigación es una actividad minuciosa y compleja que se orienta a la consecución de un fin y solución de un problema a través de un conjunto de pasos bien estructurados. Por ello, para obtener resultados positivos, se utilizó la investigación de campo ya que se estableció contacto directo con los centros educativos y donde se aplicó el instrumento de investigación; no experimental porque no se manipuló variables, solo se observó el problema para después analizarlo a través de procedimientos estadísticos; descriptiva porque se describió realidad de los contextos educativos; correlacional porque se estableció relación entre las variables de estudio.

\section{Participantes}

La muestra quedó constituida por 118 adolescentes pertenecientes a las 5 unidades educativas que dieron su consentimiento para participar en la investigación y que cursan el tercer año de bachillerato. De ellos 71 son mujeres y 47 varones, el 100\% aspira a ingresar a la Educación Superior, cuyas edades oscilan entre los 17 y 19 años.

\section{Métodos de investigación}

Los métodos de investigación son las líneas a seguir por el investigador; en este sentido, un método es un procedimiento para resolver un problema, crear teorías y sacar conclusiones.

Los métodos utilizados fueron los siguientes:

Método bibliográfico: a través de este método se fundamentó los temas más relevantes del problema en estudio.

El método analítico: permitió conocer en profundidad el problema, comprenderlo y crear conceptos suficientes para la construcción del marco teórico.

Método sintético: se utilizó para identificar y redactar las ideas principales y secundarias a fin de tener información para la construcción del marco teórico. 


\section{Calidad educativa, entorno familiar y su relación con el rendimiento académico de la enseñanza secundaria en centros educativos de la provincia de Manabí durante año 2017 -2018}

Vol. 3, núm. 2., (2019)

María José Ortiz Zurita; Jazmín Jessenia Peñafiel León; Gabriela Natalia Torres Jara; Diana Valeria Delgado Campuzano; Evelyn Mariuxi Bautista Guaranda

Método descriptivo: a través de este método se describió las características del tema en estudio, se profundizo en las variables y se conjeturó relaciones entre sí.

Método hermenéutico: permitió establecer juicios respecto a las variables a fin de obtener conclusiones acertadas para esta investigación.

Método estadístico: a través de este método se organizó la información en cuadros estaditos para poder explicar las relaciones entre las variables; para ello se utilizó el software SPSS.

Técnicas de investigación

Las técnicas utilizadas en esta investigación son: la lectura, el resumen, la observación y la encuesta.

Técnicas de investigación bibliográfica

La lectura: se utilizó para conocer los estudios anteriores a esta investigación. Además se hizo uso de la lectura exploratoria; lectura selectiva; lectura analítica; lectura crítica, las cuales permitieron entender y comparar conceptos que aporten al estudio.

El resumen: En esta investigación permitió estructurar y organizar ideas en esquemas necesarios para la construcción del marco teórico y posterior análisis de resultados.

Técnicas de investigación de campo

Observación: esta técnica permitió analizar el contexto donde se aplicó el instrumento de evaluación.

La encuesta: instrumento utilizado para obtener información de un hecho o fenómeno en estudio.

La encuesta estuvo compuesta por 161 indicadores que evalúan la calidad educativa, el entorno familiar y rendimiento académico.

Las primeras 22 preguntas evalúan la calidad de la enseñanza secundaria en aspectos como estado físico de las instalaciones, recursos y desempeño docente. 


\section{Calidad educativa, entorno familiar y su relación con el rendimiento académico de la enseñanza secundaria en centros educativos de la provincia de Manabí durante año 2017 -2018 \\ Vol. 3, núm. 2., (2019) \\ María José Ortiz Zurita; Jazmín Jessenia Peñafiel León; Gabriela Natalia Torres Jara; Diana Valeria Delgado Campuzano; Evelyn Mariuxi Bautista Guaranda}

Los indicadores que evalúan el entorno familiar están distribuidas en tres secciones:

Sección primera: 100 preguntas de los aspectos generales de la relación con ambos padres.

La sección segunda: 22 preguntas que evalúan la comunicación con ambos padres.

La sección tercera: 14 preguntas que valoran el grado de conflicto del estudiante con ambos padres.

Por último, 3 preguntas relacionadas a la percepción que tiene el estudiante del rendimiento académico.

Se precisaron de variables como: edad, tipo de centro de estudio, tipo de familia, nivel educativo de los padres e ingreso familiar.

Los estudiantes asignaron dos tipos de puntuación primero en la escala del 1 al 5, y luego en la escala del 1 al 4, de la siguiente manera: ( 1 =carece totalmente, $2=$ hay algunas carencias, $3=$ cumple regular, 4 =cumple bastante bien, 5 =cumple a la perfección); (1 =ninguna bronca, 2 =alguna bronca, 3 =bastantes broncas, 4 =muchas broncas).

\section{Procedimiento}

La presente investigación es de tipo descriptiva- interpretativa (correlacional) -explicativa, mediante el análisis crítico de las relaciones entre calidad educativa, entorno familiar y el rendimiento académico de la enseñanza secundaria, específicamente en 5 Unidades Educativas que accedieron a participar en la investigación.

La población estuvo integrada por 400 estudiantes del tercer año de bachillerato segundo semestre 2017, donde se obtuvo una muestra de 118 participantes. Para realizar esta investigación se utilizaron dos fuentes: bibliográfica y de campo. Para la bibliográfica utilicé la sugerida por la Universidad Técnica, en la Guía Didáctica previo para la obtención del título de Magister mención Pedagogía; otros textos y bibliografía disponible en la página web del internet. 


\section{Calidad educativa, entorno familiar y su relación con el rendimiento académico de la enseñanza secundaria en centros educativos de la provincia de Manabí durante año 2017 -2018}

Vol. 3, núm. 2., (2019)

María José Ortiz Zurita; Jazmín Jessenia Peñafiel León; Gabriela Natalia Torres Jara; Diana Valeria Delgado Campuzano; Evelyn Mariuxi Bautista Guaranda

Se realizó una visita previa a los centros educativos seleccionados para conocer elementos generales de los mismos, así como la viabilidad para aplicar el instrumento.

Diálogo con las autoridades del centro educativo y exposición de los motivos de la presente investigación. Luego presenté la solicitud, para obtener legalmente la autorización de parte de las autoridades y docentes a aplicarse el instrumento de investigación.

Aplicación de la encuesta. El instrumento aplicado es autoría de la Dra. C Xiomara Carrera coordinadora de la maestría de Orientación y Educación Familiar, de la Universidad Técnica Particular de Loja.

Una vez aplicados los instrumentos, se ingresó la información obtenida al SPSS para la respectiva tabulación y análisis de los resultados con el fin de generar los datos estadísticos, comparar valores y generar criterios de los aspectos investigados. 


\section{Calidad educativa, entorno familiar y su relación con el rendimiento académico de la enseñanza secundaria en centros educativos de la provincia de Manabí durante año 2017 -2018 \\ Vol. 3, núm. 2., (2019) \\ María José Ortiz Zurita; Jazmín Jessenia Peñafiel León; Gabriela Natalia Torres Jara; Diana Valeria Delgado Campuzano; Evelyn Mariuxi Bautista Guaranda}

\section{Resultados.}

Del proceso de recolección e interpretación de datos se obtuvieron los siguientes resultados

\begin{tabular}{|c|c|c|c|c|c|c|c|c|c|c|c|c|c|c|c|c|}
\hline & \multicolumn{5}{|c|}{ Fiscal } & \multicolumn{5}{|c|}{ Fisco-misional } & \multicolumn{6}{|c|}{ Privado } \\
\hline Items & 造 & 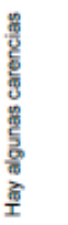 & 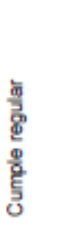 & 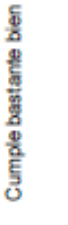 & 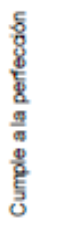 & 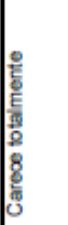 & 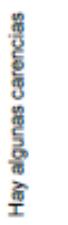 & 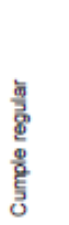 & 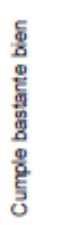 & 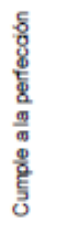 & 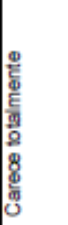 & 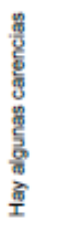 & 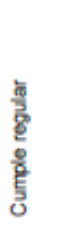 & 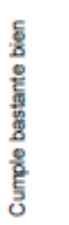 & 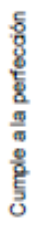 & \\
\hline $\begin{array}{l}\text { 1. Las } \\
\text { instalaciones } \\
\text { fisicas de mi } \\
\text { centro de } \\
\text { estudio se } \\
\text { encuentran en } \\
\text { buen estado }\end{array}$ & $5 \%$ & $22 \%$ & $30 \%$ & $35 \%$ & $8 \%$ & $0 \%$ & $13 \%$ & $13 \%$ & $37 \%$ & $37 \%$ & $0 \%$ & $0 \%$ & $15 \%$ & $37 \%$ & & $48 \%$ \\
\hline $\begin{array}{l}\text { El } \\
\text { equipamiento } \\
\text { de mi centro de } \\
\text { estudios parece } \\
\text { moderno }\end{array}$ & $2 \%$ & $20 \%$ & $41 \%$ & $28 \%$ & $8 \%$ & $0 \%$ & $25 \%$ & $13 \%$ & $25 \%$ & $37 \%$ & $0 \%$ & $7 \%$ & $26 \%$ & $30 \%$ & & $37 \%$ \\
\hline $\begin{array}{l}\text { 3. La } \\
\text { calefacción } \\
\text { funciona } \\
\text { correctamente }\end{array}$ & $45 \%$ & $22 \%$ & $16 \%$ & $10 \%$ & $7 \%$ & $37 \%$ & $0 \%$ & $38 \%$ & $0 \%$ & $25 \%$ & $40 \%$ & $4 \%$ & $11 \%$ & $30 \%$ & & $15 \%$ \\
\hline $\begin{array}{l}\text { 4. Ia iluminación } \\
\text { es adecuada }\end{array}$ & $4 \%$ & $14 \%$ & $28 \%$ & $33 \%$ & $21 \%$ & $0 \%$ & $0 \%$ & $50 \%$ & 0 & $50 \%$ & $0 \%$ & $4 \%$ & $11 \%$ & $30 \%$ & & $55 \%$ \\
\hline
\end{tabular}

\section{Tabla 1. Estado de las instalaciones físicas de los centros educativos}

En la tabla 1, ítem 1, el $48 \%$ de los estudiantes pertenecientes a escuelas privadas, evalúan las instalaciones físicas de su centro de estudios en buen estado; en el caso de las escuelas fisco-misionales, el 37\% emite igual criterio y en las escuelas fiscales, solo el $8 \%$ considera que las instalaciones físicas de su centro de estudios se encuentran en buen estado. Esto sugiere que las aulas, laboratorios, biblioteca, polideportivos, cafetería y baños de las escuelas privadas y fisco-misionales están en mejores condiciones que en las escuelas fiscales. Autores como Kellaghan et al. (2016) señalan que las instalaciones físicas en buen estado son indicadores de calidad educativa debido a que al contar con espacios adecuados, confortables, seguros y en óptimas condiciones se estimulan los procesos de enseñanza-aprendizaje. Con el fin de lograr una educación de calidad El Ministerio de Educación (2012) determina que los centros 


\section{Calidad educativa, entorno familiar y su relación con el rendimiento académico de la enseñanza secundaria en centros educativos de la provincia de Manabí durante año 2017 -2018}

Vol. 3, núm. 2., (2019)

María José Ortiz Zurita; Jazmín Jessenia Peñafiel León; Gabriela Natalia Torres Jara; Diana Valeria Delgado Campuzano; Evelyn Mariuxi Bautista Guaranda

educativos deben poseer condiciones de seguridad, confort, habitabilidad a fin de satisfacer los requerimientos pedagógicos del estudiantado (p. 46).

En el ítem 2 que evalúa la tenencia de equipamiento moderno el 37\% de estudiantes que pertenecen a escuelas privadas, afirman que su centro de estudios si posee equipamiento moderno; en el caso de las escuelas fisco-misionales, el 37\% son de igual opinión, mientras que, en las escuelas fiscales, solo el $8 \%$ de estudiantes considera que su escuela posee equipamiento moderno. Esto sugiere que las escuelas privadas y fisco-misionales cumplen o están próximos a cumplir con los requerimientos pedagógicos utilizados en la actualidad, a través de los cuales estimulan y agilizan el desempeño docenteestudiante en el aula. Con respecto a esto, el Instituto Nacional para la Evaluación de la Educación (2010) citado por Ministerio de Educación (2017) señala que contar con mobiliario moderno, cómodo y apropiado permite un logro efectivo de los aprendizajes ya que facilitan el involucramiento de los estudiantes durante la clase (p. 1). El Ministerio de Educación también indica que "la disponibilidad de equipamiento contribuye a que el estudiante de forma individual incremente su desempeño en el aula" (p. 2). En efecto, contar con equipamientos modernos resulta imprescindible porque proporciona el desarrollo de nuevos aprendizajes ya que ofrece escenarios innovadores sobre los cuales trabajar.

En el ítem 3 que mide funcionamiento de la calefacción, solo el 15\% de estudiantes que pertenecen a escuelas privadas evalúan de perfecto el funcionamiento de la misma, en el caso de las escuelas fisco-misionales, el 25\% son de igual opinión. Con relación a las escuelas fiscales, solo el 7\% de los estudiantes señala que la calefacción funciona correctamente. Esto sugiere que las escuelas fiscomisionales presentan mejores condiciones donde se efectúa el aprendizaje en relación con las escuelas privadas y fiscales. De modo general y considerando los valores obtenidos se observa que las escuelas fiscales, fisco-misionales y privadas presentan carencias en cuanto a equipos técnico - ambientales (aire acondicionado-ventiladores). Respecto a esto, Vélez et al. (1994) en su estudio indica resultados positivos entre la infraestructura básica (por ejemplo, electricidad, agua y mobiliario) y el rendimiento académico. Steve Higgins (2005) citado en Abend et al., (2009) en su revisión literaria señala que "los ambientes de aprendizaje extremadamente pobres tienen un efecto negativo en los estudiantes y personal docente (p. 18)". En efecto, la ausencia de equipos técnicos-ambientales (aire acondicionado, ventiladores) dificultan la tarea docente y estudiante debido a que laborar en ambientes poco adecuados (bajas o altas temperaturas) disminuye la actividad perceptiva y ralentiza el aprendizaje. 


\section{Calidad educativa, entorno familiar y su relación con el rendimiento académico de la enseñanza secundaria en centros educativos de la provincia de Manabí durante año 2017 -2018}

Vol. 3, núm. 2., (2019) María José Ortiz Zurita; Jazmín Jessenia Peñafiel León; Gabriela Natalia Torres Jara; Diana Valeria Delgado Campuzano; Evelyn Mariuxi Bautista Guaranda

En el ítem 4, para el 55\% de estudiantes que pertenecen escuelas privadas, la iluminación de su centro de estudios es adecuada; en el caso de las escuelas fisco-misionales el 50\% son de igual opinión. En relación con las escuelas fiscales, solo el $21 \%$ de los estudiantes considera que la iluminación de su centro educativo es adecuada. Esto sugiere que las escuelas privadas y fisco-misionales cuentan con aulas debidamente iluminadas que estimulan los procesos perceptivos que influyen en el aprendizaje. Por el contrario, en las escuelas fiscales se observa un bajo porcentaje que indica que las aulas están debidamente iluminadas. Condiciones físicas que pueden provocar fatiga, monotonía, desmotivación y dificultades en los procesos de percepción y atención en el alumnado.

Con respecto a esto, Mark Schneider como se citó en Abend et al., (2009) al examinar 6 categorías que afectan los resultados académicos concluye que la configuración del espacio, el ruido, calor, frío, luz y calidad del aire influyen en el rendimiento tanto de los estudiantes como de los maestros (p. 18). El estudio realizado por la Universidad de Nebrija (2012) en el Colegio Público Francisco de Quevedo Madrid, al utilizar el sistema de alumbrado SCHOOLVISIÓN concluyó que la iluminación tiene impacto positivo en los alumnos con dificultades, debido a que aumenta la agudeza visual y la concentración de los estudiantes. En efecto, una adecuada iluminación proporciona un ambiente propicio que estimula las habilidades perceptivas y favorece los aprendizajes.

Como tendencia se obtuvo que las escuelas privadas y fisco-misionales presentan de modo general, según la evaluación de los estudiantes, mejores condiciones físicas y de infraestructura que las escuelas fiscales. En cuanto a los indicadores evaluados se evidencia que las escuelas fiscales aproximadamente el $50 \%$ de los encuestados evalúan por debajo de la media las condiciones de las instalaciones, equipamiento, calefacción e iluminación. Los aspectos con evaluaciones más desfavorables en los 3 tipos de centro educativo son la calefacción y el uso de equipamiento moderno.

Los contenidos evaluados en esta tabla son de gran importancia ya que inciden en el éxito de los estudiantes, así como en su proceso académico y posterior adaptación a la vida universitaria. Como apuntan los diversos estudios consultados, instalaciones físicas en óptimas condiciones, contribuyen significativamente al desempeño docente-estudiante ya que crea ambientes propicios que estimulan los procesos pedagógicos mejorando el rendimiento académico de los estudiantes. 


\section{Calidad educativa, entorno familiar y su relación con el rendimiento académico de la enseñanza secundaria en centros educativos de la provincia de Manabí durante año 2017 -2018}

Vol. 3, núm. 2., (2019)

María José Ortiz Zurita; Jazmín Jessenia Peñafiel León; Gabriela Natalia Torres Jara; Diana Valeria Delgado Campuzano; Evelyn Mariuxi Bautista Guaranda

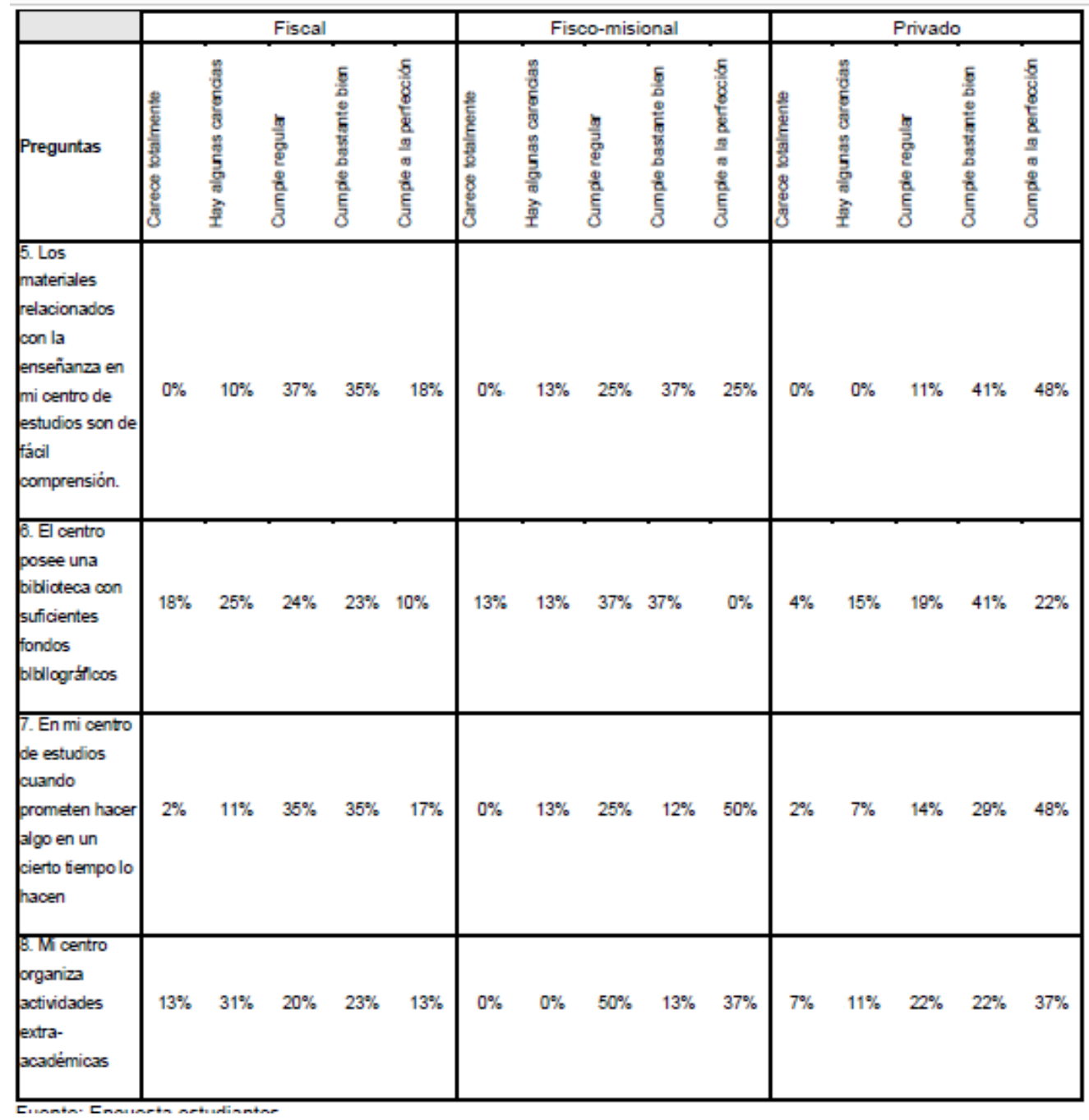

Tabla 2. Aspectos educativos de los centros de estudio

En la tabla 2, ítem 5, para el $48 \%$ de estudiantes que pertenecen a escuelas privadas, los materiales relacionados a la enseñanza son de fácil comprensión; en el caso de las escuelas fiscomisionales, el 25\% son de igual opinión. En relación con las escuelas fiscales, solo el 18\% de estudiantes considera que los materiales son de fácil comprensión. Esto sugiere que las escuelas privadas brindan materiales pedagógicos más adaptados a las capacidades, habilidades y características propias del período etario de los estudiantes; evidencian altos niveles de preparación del profesorado y dedicación de los mismos a la elaboración de materiales de apoyo a la docencia que facilitan el cumplimiento de los 


\section{Calidad educativa, entorno familiar y su relación con el rendimiento académico de la enseñanza secundaria en centros educativos de la provincia de Manabí durante año 2017 -2018}

Vol. 3, núm. 2., (2019) María José Ortiz Zurita; Jazmín Jessenia Peñafiel León; Gabriela Natalia Torres Jara; Diana Valeria Delgado Campuzano; Evelyn Mariuxi Bautista Guaranda

objetivos en cada materia, así como la existencia de condiciones materiales y de equipamiento para la confección de dichos materiales. Con respecto a esto, Pernilla (2013) señala que cuando algo es considerado interesante, aumenta la dedicación, y, consecuentemente, es importante que los contenidos y las actividades de la enseñanza sean interesantes para los estudiantes (pág. 7). Skolverket (como se citó en Pernilla, 2013) esgrime la importancia de que el material educativo genere el interés (pág. 1). Gonzales èt al. (2014), en su estudio determinó que el material didáctico que tiene en cuenta la motivación, la fijación de los conceptos y el refuerzo, influye en el aprendizaje signficativo (p. 8). Es necesario que los recursos didácticos que brindan las instituciones educativas sean accesibles a la comprensión y estimulen la motivación y generen el aprendizaje de los estudiantes.

En el ítem 6, para el 37\% de estudiantes que pertenecen escuelas fisco-misionales, sus centros poseen bibliotecas con suficientes recursos bibliográficos; en el caso de las escuelas privadas el 22\% son de igual opinión. En relación con las escuelas fiscales, solo el 10\% de estudiantes considera que sus centros educativos poseen biblioteca con suficientes recursos bibliográficos. Esto sugiere que existen mayores recursos para la formación en las escuelas fisco-misionales y que se presenta en menor proporción en las escuelas fiscales y privadas. La biblioteca es un recurso del cual no pueden prescindir los centros educativos y que se relaciona con los procesos enseñanza-aprendizaje, existiendo otros elementos como la estimulación del profesorado para que se asista a la biblioteca para reforzar las habilidades investigativas y hábitos como la lectura, y retomar la importancia del conocimiento de autores clásicos antológicos en el fomento de una cultura general. Para López Pérez (2015) "la biblioteca como herramienta básica en la formación lectora, favorece el aprendizaje en todas las áreas, ciclos y etapas, fomentando hábitos de lectura, habilidades de información, formación y de autonomía" (p. 3). Tejeda (2006) señala que "la biblioteca desarrolla en los alumnos, habilidades de aprendizaje para toda la vida, estimula sus capacidades imaginativas y les ayuda a asumir un rol como ciudadanos responsables" (pág. 1). En efecto, este recurso impulsa el desarrollo y variedad de habilidades cognitivas y de análisis necesarias para un desempeño exitoso, además de fomentar la formación de una cultura general integral en el estudiantado, en consonancia con lo que afirma Vélez et al. (1994), "el acceso a libros de texto y otro tipo de material instructivo es importante ya que incrementa el rendimiento académico de los estudiantes" (p. 12). 


\section{Calidad educativa, entorno familiar y su relación con el rendimiento académico de la enseñanza secundaria en centros educativos de la provincia de Manabí durante año 2017 -2018}

Vol. 3, núm. 2., (2019)

María José Ortiz Zurita; Jazmín Jessenia Peñafiel León; Gabriela Natalia Torres Jara; Diana Valeria Delgado Campuzano; Evelyn Mariuxi Bautista Guaranda

En el ítem 7, para el 50\% de estudiantes que pertenecen a escuelas fisco-misionales, sus centros educativos si cumplen con la planificación; en el caso de las escuelas privadas, el $48 \%$ son de igual opinión. En relación con las escuelas fiscales, solo el 17\% considera que sus centros si cumplen con la planificación. Esto sugiere que existe mayor y mejores niveles de comunicación y gestión pedagógica en las escuelas fisco-misionales y privadas que en las escuelas fiscales. Con respecto a esto, la planificación es una actividad fundamental que permite alcanzar objetivos, mejorar los procesos y contribuir con el mejoramiento de la educación. Es necesaria porque a través de ella se consiguen aprendizajes en un determinado tiempo, evitando en el docente la improvisación innecesaria. Para Venegas Jiménez (2004) la planificación de la educación es "el instrumento que permite conocer, prever y actuar sobre una realidad determinada (...)” (p. 104). En efecto, una buena planificación optimiza y potencia los procesos de enseñanza-aprendizaje, refuerza el vínculo profesor- alumno y contribuye a organizar de manera coherente el cumplimiento de los objetivos en los tiempos esperados, procurando una mejor intervención por parte de los docentes y un mejor desempeño de los estudiantes.

En el ítem 8, para el $37 \%$ de estudiantes que pertenecen a escuelas a escuelas privadas, sus centros educativos organizan actividades extra-académicas; en el caso de las escuelas fisco-misionales, el $37 \%$ son de igual opinión. En relación con las escuelas fiscales, solo el 13\% considera que sus centros educativos organizan actividades extra-académicas. Esto sugiere que las escuelas privadas y fiscomisionales tienden a preocuparse más por la formación continua e integral de los estudiantes, donde se combinan actividades formales de la malla curricular con otras de conocimiento general las cuales promueven la interacción entre los pares, además de desarrollar acciones de aprendizaje lúdico y buen uso del tiempo libre, actividades vinculadas con la cultura, el arte, el deporte, al aire libre, entre otras, lo cual contribuye a una formación general y para la vida de cada estudiante y a la excelencia académica del claustro.

Las actividades extra-académicas representan un recurso de apoyo y refuerzo a la formación recibida en los salones de clase. El IES "Valle del Kas" Citado en Serón, (2003) recoge en el RRI que aparece en su agenda del curso 2003 - 2004 una declaración de principios y reglamentación de las actividades complementarias y extraescolares y de las que dice "representan un eficaz instrumento de intervención educativa que prolonga la actividad docente más allá del espacio y del tiempo habituales” (p. 16). Un estudio realizado por Carmona Rodríguez, Sánchez Delgado, \& Bakieva, (2011) sobre 


\section{Calidad educativa, entorno familiar y su relación con el rendimiento académico de la enseñanza secundaria en centros educativos de la provincia de Manabí durante año 2017 -2018}

Vol. 3, núm. 2., (2019)

María José Ortiz Zurita; Jazmín Jessenia Peñafiel León; Gabriela Natalia Torres Jara; Diana Valeria Delgado Campuzano; Evelyn Mariuxi Bautista Guaranda

“Actividades extraescolares y rendimiento académico: diferencias en auto-concepto y género" señaló que los que realizaban las actividades extraescolares de tipo académico como idiomas e informática obtuvieron mejores resultados en todas las materias de rendimiento académico (p. 1). En efecto, las actividades extra-académicas contribuyen a un mejor desempeño del estudiante, estimulando habilidades cognitivas, la adquisición de conocimientos y favoreciendo los aprendizajes en el aula.

De modo general en esta tabla se aprecia que los ítems con mayores dificultades son la actualización del fondo bibliográfico de las bibliotecas y el número de actividades extra académicas que se realizan, en los tres tipos de instituciones. Se aprecia que las escuelas fiscales son las más afectadas, pues presentan mayores carencias en relación a las escuelas privadas y fisco-misionales en aspectos como: recursos bibliográficos, didácticos y actividades extra-académicas. Aspectos que resultan imprescindibles en la formación, debido a que contribuyen a la adquisición de destrezas y habilidades cognitivas necesarias para el desempeño del estudiante en el aula.

\begin{tabular}{|c|c|c|c|c|c|c|c|c|c|c|c|c|c|c|c|}
\hline & \multicolumn{5}{|c|}{ Fiscal } & \multicolumn{5}{|c|}{ Fisco-misional } & \multicolumn{5}{|c|}{ Privado } \\
\hline Preguntas & 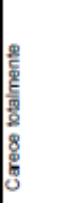 & 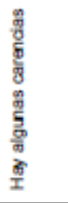 & 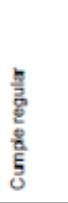 & 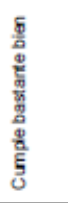 & 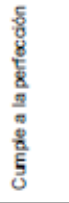 & 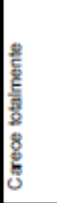 & 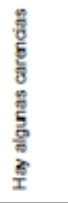 & 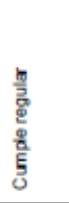 & 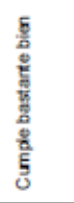 & 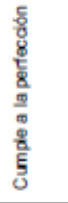 & 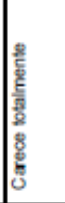 & 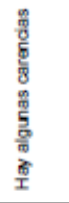 & 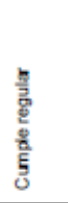 & 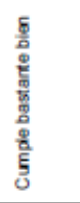 & 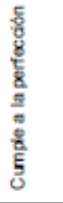 \\
\hline $\begin{array}{l}\text { El profesorado } \\
\text { iene } \\
\text { conocimientos } \\
\text { suficientes para } \\
\text { contestar a las } \\
\text { cuestiones de los } \\
\text { alumnos }\end{array}$ & $2 \%$ & $8 \%$ & $19 \%$ & $43 \%$ & $28 \%$ & $0 \%$ & $12 \%$ & $13 \%$ & $25 \%$ & $50 \%$ & $4 \%$ & $0 \%$ & $7 \%$ & $41 \%$ & $48 \%$ \\
\hline $\begin{array}{l}\text { 10. Las casses } \\
\text { combinan } \\
\text { aspectos téricos y } \\
\text { prácticos }\end{array}$ & $0 \%$ & $8 \%$ & $28 \%$ & $41 \%$ & $23 \%$ & $0 \%$ & $12 \%$ & $13 \%$ & $25 \%$ & $50 \%$ & $0 \%$ & $0 \%$ & $19 \%$ & $48 \%$ & $33 \%$ \\
\hline $\begin{array}{l}\text { 11. Los profesores } \\
\text { fomentan el interés } \\
\text { por las asignaturas } \\
\text { que imparten entre } \\
\text { os alumnos }\end{array}$ & $2 \%$ & $10 \%$ & $35 \%$ & $37 \%$ & $16 \%$ & $0 \%$ & $12 \%$ & $13 \%$ & $50 \%$ & $25 \%$ & $3 \%$ & $0 \%$ & $19 \%$ & $37 \%$ & $41 \%$ \\
\hline $\begin{array}{l}\text { 12. los profesores } \\
\text { combinan medios } \\
\text { de enseñanza } \\
\text { radicional con } \\
\text { modemos }\end{array}$ & $4 \%$ & $15 \%$ & $22 \%$ & $23 \%$ & $36 \%$ & $0 \%$ & $0 \%$ & $38 \%$ & $12 \%$ & $50 \%$ & $0 \%$ & $4 \%$ & $18 \%$ & $19 \%$ & $50 \%$ \\
\hline
\end{tabular}

Tabla 3. Desempeño docente 


\section{Calidad educativa, entorno familiar y su relación con el rendimiento académico de la enseñanza secundaria en centros educativos de la provincia de Manabí durante año 2017 -2018}

Vol. 3, núm. 2., (2019)

María José Ortiz Zurita; Jazmín Jessenia Peñafiel León; Gabriela Natalia Torres Jara; Diana Valeria Delgado Campuzano; Evelyn Mariuxi Bautista Guaranda

En la tabla 3, ítem 9 los profesores poseen suficientes conocimientos para contestar sus dudas, más del 65\% de los encuestados evalúan este ítem de: cumplen bastante y a la perfección la aclaración de dudas. Esto sugiere que existe una buena preparación y formación profesoral, experiencia amplia en el magisterio y maestría pedagógica para la impartición de clases, además de promover la gestión del conocimiento en las aulas. Según Artunduaga (2014) entre las variables que inciden en el rendimiento académico están: la formación y experiencia del docente (p. 273). Con respecto a esto Vélez et al., (1994), señala que la experiencia y formación docente están positivamente relacionadas con el rendimiento debido a que de ellas depende en gran medida la calidad de la educación. En efecto, brindan al docente elementos científicos, técnicos y humanísticos que contribuyen a un mejor desempeño en el aula.

En el ítem 10 las clases combinan aspectos teóricos y prácticos, más del 50\% de los estudiantes encuestados en todas las instituciones, evalúan estos ítems de: se cumple bastante y cumple a la perfección. Los aspectos teóricos se consolidan con los prácticos y viceversa, por lo tanto, una educación que no considere estos componentes de forma relacional cae en un reduccionismo que atentan contra los procesos de aprendizaje del estudiante, siendo este binomio uno de los más difíciles de cumplir en la clase; esta vinculación teoría- práctica estimula los procesos del pensamiento, fortalece las habilidades cognitivas de los estudiantes y contribuye a la formación integral del conocimiento en el alumnado, además de una construcción del conocimiento colectiva donde los estudiantes son protagonistas en la clase a la vez que responsables de su proceso de aprender . López Aguilar \& Sánchez Dorantes (2010) señalan la importancia de utilizar un enfoque constructivista, que propicie el papel activo del estudiante, la interacción con los compañeros y la vinculación de los contenidos con sus experiencias de vida (p. 1). Vélez et al., (1994), indica que los métodos de enseñanza activa son más efectivos que los pasivos. Ambos autores coinciden en la importancia de los aspectos prácticos debido a que permiten consolidar el conocimiento en aprendizajes significativos.

En el ítem 11: Los profesores fomentan el interés por las asignaturas que imparten entre los alumnos. Más del 50\% de la muestra total evalúa estos ítems de: se cumple bastante y cumple a la perfección. Esto sugiere un alto nivel de preparación del profesorado, y la magistralidad en la impartición de las clases incluyendo habilidades pedagógicas que invitan a la participación de los estudiantes y la búsqueda activa del conocimiento, vínculo teoría- práctica, además de las posturas y actitudes que asumen los maestros en el contexto aúlico. Es importante señalar que las actitudes de los profesores favorecen la 


\section{Calidad educativa, entorno familiar y su relación con el rendimiento académico de la enseñanza secundaria en centros educativos de la provincia de Manabí durante año 2017 -2018}

Vol. 3, núm. 2., (2019) María José Ortiz Zurita; Jazmín Jessenia Peñafiel León; Gabriela Natalia Torres Jara; Diana Valeria Delgado Campuzano; Evelyn Mariuxi Bautista Guaranda

promoción de buenas situaciones de enseñanzas (De Souza Barros \& F. Elia, 2014). Creando un clima donde el estudiante puede intervenir sin miedos. Por ello, García \& Reyes (2014) señalan que para que haya acierto en el proceso de aprender, la relación entre el maestro y alumnos debe estar basada en la atención, el respeto, el compromiso y el agrado de recibir la educación y de dar la enseñanza (2014, p. 282). Otro aspecto a considerar y que es parte de la tarea docente es la motivación que debe dar al estudiante que no debe ser tan solo lo referente a la asignatura, sino a las diferentes áreas del conocimiento. Esta y otras formas de relación, son importantes debido a que contribuyen a la creación de ambientes educativos que estimulan y favorecen el aprendizaje.

En el ítem 12 las clases combinan medios de enseñanza tradicional con modernos, más del $60 \%$ de los encuestados evalúa este ítem de: se cumple bastante y cumple a la perfección. Esto sugiere que las escuelas se preocupan por incluir en sus procesos pedagógicos herramientas actuales que faciliten y promuevan nuevos aprendizajes; pues permiten la facilitación del proceso, a través de objetos reales, sus representaciones sirven de apoyo material para la apropiación del contenido, complementan el método y el cumplimiento de los objetivos propuestos por el docente. Estimulan el proceso de comunicación, favorece la formación de hábitos, habilidades y destrezas así como normas de conductas en los estudiantes. Motivan el aprendizaje y aumentan los niveles de concentración.

Peréz Gutiérrez \& Florido Bacallao (2003) señalan que el internet facilita el aprendizaje colaborativo, provee una gran cantidad de recursos educativos, mejora las vías de comunicación y satisface las necesidades de aprendizajes de los estudiantes (p. 5). Además, contribuye al desarrollo de habilidades de estudio como: alfabetización tecnológica, habilidades de búsqueda, selección, competencias lingüísticas, interés y motivación. En efecto, utilizar estrategias modernas en la enseñanza secundaria configura un nuevo marco para el desarrollo y apropiación del conocimiento debido a que mejora y agiliza los aprendizajes. Por lo tanto, los centros educativos deben considerar combinar el uso de los medios tradicionales con los modernos a fin de lograr calidad en la educación.

A pesar de que los porcentajes son ligeramente más bajos en las escuelas fiscales que las privadas y fisco-misionales en cuanto al desempeño docente. De modo general se puede afirmar que los docentes están muy bien preparados en especial lo referido a la maestría pedagógica y el dominio de los conocimientos propios de las materias que imparten. Es importante señalar que este es un tema central en el proceso de enseñanza- aprendizaje debido a que la pedagogía, los métodos y actitudes que asume el 


\section{Calidad educativa, entorno familiar y su relación con el rendimiento académico de la enseñanza secundaria en centros educativos de la provincia de Manabí durante año 2017 -2018}

Vol. 3, núm. 2., (2019)

María José Ortiz Zurita; Jazmín Jessenia Peñafiel León; Gabriela Natalia Torres Jara; Diana Valeria Delgado Campuzano; Evelyn Mariuxi Bautista Guaranda

docente son fundamentales porque favorecen la adquisición de destrezas y habilidades que necesita el estudiante para su desempeño académico y para la vida.

\begin{tabular}{|c|c|c|c|c|c|c|c|c|c|c|c|c|c|c|c|}
\hline & \multicolumn{5}{|c|}{ Nuclear } & \multicolumn{5}{|c|}{ Extensa } & \multicolumn{5}{|c|}{ Monoparenta } \\
\hline Ittems & $\begin{array}{l}\mathscr{g} \\
\frac{1}{2} \\
\end{array}$ & 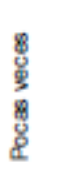 & 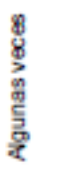 & 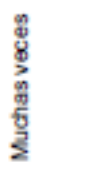 & $\begin{array}{l}\text { ֻ. } \\
\text { है } \\
\text { ळ }\end{array}$ & $\frac{8}{2}$ & $\begin{array}{l}8 \\
8 \\
\mathscr{g}^{2} \\
8 \\
8 \\
8\end{array}$ & $\begin{array}{l}\frac{8}{8} \\
\frac{8}{2} \\
\frac{8}{8} \\
\frac{2}{5} \\
\frac{5}{2}\end{array}$ & 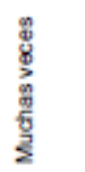 & $\begin{array}{l}\frac{\mathscr{U}}{\mathrm{g}} \\
\text { ळ }\end{array}$ & $\begin{array}{l}\frac{g}{2} \\
\frac{2}{2} \\
\end{array}$ & 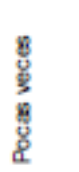 & $\begin{array}{l}8 \\
8 \\
\frac{8}{2} \\
\frac{1}{2} \\
\frac{2}{5} \\
\frac{9}{2}\end{array}$ & 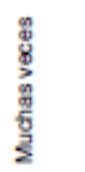 & $\begin{array}{l}\text { ֻั. } \\
\text { हू } \\
\text { कू }\end{array}$ \\
\hline $\begin{array}{l}\text { 13. Dedican } \\
\text { tiempo hablar } \\
\text { conmigo }\end{array}$ & $3 \%$ & $20 \%$ & $27 \%$ & $30 \%$ & $20 \%$ & $7 \%$ & $31 \%$ & $24 \%$ & $35 \%$ & $8 \%$ & $57 \%$ & $0 \%$ & $14 \%$ & $22 \%$ & $7 \%$ \\
\hline $\begin{array}{l}\text { 14. son } \\
\text { carinosola } \\
\text { conmigo }\end{array}$ & $11 \%$ & $11 \%$ & $21 \%$ & $25 \%$ & $32 \%$ & $4 \%$ & $15 \%$ & $42 \%$ & $24 \%$ & $15 \%$ & $43 \%$ & $29 \%$ & $7 \%$ & $0 \%$ & $21 \%$ \\
\hline $\begin{array}{l}\text { 15. me permiten } \\
\text { discutir las } \\
\text { normas cuando } \\
\text { creo que no son } \\
\text { fustas }\end{array}$ & $10 \%$ & $28 \%$ & $31 \%$ & $17 \%$ & $14 \%$ & $12 \%$ & $15 \%$ & $15 \%$ & $23 \%$ & $35 \%$ & $57 \%$ & $14 \%$ & $14 \%$ & $0 \%$ & $15 \%$ \\
\hline $\begin{array}{l}\text { 18. me exigen } \\
\text { respeto } \\
\text { absoluto a su } \\
\text { autoridad }\end{array}$ & $4 \%$ & $8 \%$ & $13 \%$ & $30 \%$ & $45 \%$ & $12 \%$ & $8 \%$ & $31 \%$ & $27 \%$ & $22 \%$ & $43 \%$ & $7 \%$ & $0 \%$ & $36 \%$ & $14 \%$ \\
\hline
\end{tabular}

Tabla 4. Clima familiar y tipo de familia

En la tabla 7, ítem 13, para el 20\% de estudiantes que pertenecen a la familia nuclear, sus padres dedican tiempo hablar con ellos; en el caso de la familia extensa con $8 \%$ y familia monoparental con $7 \%$ son de igual opinión. Esto sugiere que la familia nuclear biparental brinda mejores condiciones para establecer un diálogo abierto con los hijos que favorece el desarrollo de estos, en sus diferentes áreas. Sin embargo podemos afirmar que el porcentaje es bajo en sentido general y por tanto el uso del tiempo libre para compartir en familia es escaso, lo que dificulta los procesos de comunicación, las interacciones entre 


\section{Calidad educativa, entorno familiar y su relación con el rendimiento académico de la enseñanza secundaria en centros educativos de la provincia de Manabí durante año 2017 -2018}

Vol. 3, núm. 2., (2019) María José Ortiz Zurita; Jazmín Jessenia Peñafiel León; Gabriela Natalia Torres Jara; Diana Valeria Delgado Campuzano; Evelyn Mariuxi Bautista Guaranda

los miembros son escasas y por tanto el nivel de conocimiento que poseen los padres acerca de sus hijos. Respecto a esto Leal Gonzáles (1994) señala que uno de los factores que discurre en el rendimiento académico es la comunicación familiar (p. 23). Que según Guzmán Arteaga \& Pacheco Lora (2014), es el vehículo indispensable en el interior de la familia que permite el crecimiento de sus miembros. Así mismo, en el estudio realizado por estos autores confirmaron que el acompañamiento y la comunicación que los estudiantes tengan en el hogar están asociados con el rendimiento académico. Por lo tanto, la comunicación que los padres establecen con sus hijos es fundamental ya que permite orientarlos en los ámbitos personal, académico y social; además, favorece los procesos interactivos brindando soporte para que los hijos se desarrollen adecuadamente y se adapten a diversas situaciones, entre esta las educativas.

En el ítem 14, para 32\% de estudiantes que pertenecen a la familia nuclear, sus padres son cariñosos; en el caso de la familia monoparental con $21 \%$ y familia extensa con $15 \%$ son de igual opinión. Esto sugiere que los estudiantes de familias nucleares reciben más afecto que los de familias extensas y monoparentales, no obstante los porcientos tienden a ser bajos en todos los tipos de familia, quedando al descubierto una brecha en las funciones de la familia como grupo primario de socialización donde se generan: Normas y patrones de conducta, sostén emocional, protección, satisfacción de necesidades materiales y espirituales. A este respecto, Bernal, Rivas, \& Urpí (2012) señalan que el afecto es la capacidad de los padres para responder con sensibilidad a las necesidades de los hijos exteriorizando cariño, calidez y aceptación (p. 96). Autores como Ramírez (2005) hablan de estilos de crianza en donde destacan la importancia de la práctica del estilo democrático donde los padres son afectuosos, refuerzan el comportamiento, evitan el castigo y son sensibles a las peticiones de los hijos, proporcionando el clima idóneo donde se refuerzan los aprendizajes positivos. Por lo tanto, el grado en que los padres brinden afecto a los hijos determinará el desarrollo emocional de estos, que facilitará un clima idóneo para los aprendizajes y su adaptación al contexto.

En el ítem 15, para el 35\% de estudiantes que pertenecen a la familia extensa, sus padres permiten discutir las normas cuando no son justas; en el caso de la familia monoparental con $15 \%$ y nuclear con $14 \%$ son de igual opinión. Esto sugiere que las relaciones establecidas en la familia extensa son más flexibles en cuanto a las normas en relación a las familias monoparental y nuclear. Sin embargo, se observa mayor control parental en la familia nuclear y monoparental. No obstante se deduce tendencia a la rigidez en la dinámica familiar y estilos autoritarios, que generan entornos con dificultades en los 


\section{Calidad educativa, entorno familiar y su relación con el rendimiento académico de la enseñanza secundaria en centros educativos de la provincia de Manabí durante año 2017 -2018}

Vol. 3, núm. 2., (2019)

María José Ortiz Zurita; Jazmín Jessenia Peñafiel León; Gabriela Natalia Torres Jara; Diana Valeria Delgado Campuzano; Evelyn Mariuxi Bautista Guaranda

procesos de comunicación, relaciones interpersonales fuertemente jerarquizadas; poca capacidad de expresar afectos positivos y sentimientos, pocos recursos personales para expresar los desacuerdos sin carácter violento y para negociar. Poca capacidad empática de los miembros. Tendencia a un pobre respeto y comprensión por los problemas de los otros miembros de la familia. Todo lo anterior influye de manera negativa en el rendimiento académico de los hijos.

A este respecto Oliva y Parra (2012) mencionan que uno de los factores relacionados al rendimiento académico es el grado de control o monitorización que se ejerce sobre la conducta de los hijos, en efecto, las normas son necesarias debido a que permiten a los jóvenes adaptarse a los diferentes contextos y al mismo tiempo impiden la aparición de conductas inadecuadas. Los Autores Bernal et al. (2012), indican la importancia que tiene el control parental sobre los hijos ya que contribuye en el desarrollo hacia la madurez, en el sentido, que permite acomodarse a las normas y criterios que les indiquen (p. 97). Por lo tanto, la puesta en práctica de pautas y normas de comportamiento resulta importante pues contribuye a la formación y consolidación de comportamientos adecuados que facilitan la adaptación a los diferentes contextos.

En el ítem 16, para el $45 \%$ de estudiantes que pertenecen a la familia nuclear, sus padres exigen respeto absoluto a su autoridad; en el caso de la familia extensa con $22 \%$ y familia monoparental con $14 \%$ son de igual opinión. Se observa como tendencia, una pobreza en los recursos comunicacionales (comunicación directa, abierta y flexible) y de negociación para con los hijos, no hay claridad en los límites establecidos al interior de la familia, estilos educativos autoritarios lo cual repercute en el funcionamiento familiar y en el clima sociopsicológico que prevalece, además de limitar el crecimiento sano de sus miembros.

Respecto a esto, Baumrind como se citó en Izzedin \& Pantoja (2009) señala que el estilo autoritario es rígido pues favorece la disciplina en demasía, dándole importancia a los castigos limitando la autonomía y la creatividad. Los autores Bernal et al. (2012), señalan que los resultados de aplicar el estilo autoritario ocasiona personas menos afectivas, desapegadas emocionalmente, retraimiento y timidez, desconfianza, insatisfacción (p. 101) El estudio realizado por Tilano, Henao, \& Restrepo (2009) señalaron que estilos rígidos propician un desempeño académico deficitario. En efecto, aplicar en demasía el estilo autoritario dificulta el desarrollo emocional, social y cognitivo del adolescente. Por el contrario, la puesta en práctica de normas claras proporciona un contexto en el cual los adolescentes pueden 


\section{Calidad educativa, entorno familiar y su relación con el rendimiento académico de la enseñanza secundaria en centros educativos de la provincia de Manabí durante año 2017 -2018}

Vol. 3, núm. 2., (2019)

María José Ortiz Zurita; Jazmín Jessenia Peñafiel León; Gabriela Natalia Torres Jara; Diana Valeria Delgado Campuzano; Evelyn Mariuxi Bautista Guaranda

interactuar sin miedo al error, favoreciendo un clima positivo que estimula la comunicación y el desarrollo de habilidades.

De modo general hay una tendencia en las familias - independientemente de su clasificación- a presentar dificultades en los procesos comunicativos, y un cumplimiento parcial de las funciones familiares en específico lo referido a: generadora de afectos y sostén emocional; así como la prevalencia de estilos educativos rígidos y autoritarios.

\section{Conclusiones.}

Las Unidades Educativas participantes en el estudio poseen las condiciones apropiadas de infraestructura y el recurso humano capacitado, necesarios para generar un proceso docente educativo de calidad.

Existe una estrecha relación entre las variables calidad educativa y el entorno familiar (tipología de familia según su tamaño y nivel de funcionamiento) las características de ambos elementos son determinantes en el rendimiento académico de los estudiantes, según la percepción que poseen los estudiantes. En el presente estudio, los sujetos pertenecientes a familia nucleares y que asisten a escuelas privadas y fisco-misionales obtuvieron un rendimiento académico más alto.

Las variables calidad educativa y entorno familiar tienden a influir de modo directo y determinante en el rendimiento académico de los adolescentes en estudio. Entre los factores de mayor incidencia están: nivel económico de la familia, comunicación, desempeño docente, normas, autoridad y estilos educativos empleados por padres y maestros.

Las variables referidas al estado de las instalaciones físicas, el desempeño docente y la gestión pedagógica inciden significativamente en el rendimiento académico de los estudiantes. Siendo Los centros educativos privados y fisco-misionales los que presentan una situación más favorable en las mismas. 


\section{Calidad educativa, entorno familiar y su relación con el rendimiento académico de la enseñanza secundaria en centros educativos de la provincia de Manabí durante año 2017 -2018}

Vol. 3, núm. 2., (2019)

María José Ortiz Zurita; Jazmín Jessenia Peñafiel León; Gabriela Natalia Torres Jara; Diana Valeria Delgado Campuzano; Evelyn Mariuxi Bautista Guaranda

\section{Bibliografía.}

Bautista Sánchez, M. G., Martínez Moreno, A. R., \& Hiracheta Torres, R. (2014). El uso de material didáctico y las tecnologías de información y comunicación (TIC's) para mejorar el alcance académico. Facultad de Ingeniería Mecánica y Eléctrica de la Universidad Autónoma de Nuevo León, 183-194.

García Rangel, E. G., García Rangel, A. K., \& Reyes Angulo, J. A. (2014). Relación maestro alumno y sus implicaciones en el aprendizaje. RAXIMHAI, 279-290.

López Aguilar, N., \& Sánchez Dorantes, L. (2010). El aburrimiento en clases. Procesos Psicológicos y Sociales, 1-43.

Abend, Almeida, R., Valssasina Heitor, T., Ornstein, S., \& Thomson, K. (2009). Estudio Piloto Internacional Evaluación de la Calidad en Espacios Educativos Manual del Usuario.

Acevedo Quiroz, L. H. (julio-diciembre de 2011). El concepto de familia hoy. Franciscanum. Revista de las ciencias del espíritu, LIII (156), 149-170.

Alberdi, I. (1997). La familia. Convergencia y divergencia de los modelos familiares españoles en el entorno europeo. En Política y Sociedad (págs. 73-94). Madrid.

Ángel, H., Oliva, A., \& Pertegal, M.-Á. (2012). Variables familiares y rendimiento académico en la adolescencia. Estudios de Psicología, 33(1).

Ariza Dau, M., Ramos Ruíz, J. L., \& Rodriguez Albor, G. (2012). Calidad institucional y rendimiento académico. Perfiles educativos, 36(143), 1-20.

Barbera, C. G. (2003). Factores determinantes del bajo rendimiento académico en educación secundaria. Madrid: Universidad Complutense de Madrid.

Barca Lozano, A., Porto Rioboo, A., Brenlla Blanco, J., Morán Fraga, H., \& Barca Enríquez, E. (2007). Contextos familiares y rendimiento escolar en el alumnado de educacion secundaria. INFAD Revista de Psicología (2), 197-218.

Beneyto Sánchez, S. (2015). Entorno Familiar y Rendimiento Académico. ÁREA DE INNOVACIÓN Y DESARROLLO, S.L., 1-96.

Bernal, A., Rivas, S., \& Urpí, C. (2012). Educación familiar: Infancia y adolescencia. Madrid: Ediciones Pirámide.

Berzosa Alonso-Martinez, J., Regodón Fuertes, C., \& Santamaría de Gracia, L. (2011). La familia un concepto siempre moderno. Madrid: Centro Regional de Formación e Investigación en Servicios Sociales "Beatriz Galindo". 


\section{Calidad educativa, entorno familiar y su relación con el rendimiento académico de la enseñanza secundaria en centros educativos de la provincia de Manabí durante año 2017 -2018}

Vol. 3, núm. 2., (2019)

María José Ortiz Zurita; Jazmín Jessenia Peñafiel León; Gabriela Natalia Torres Jara; Diana Valeria Delgado Campuzano; Evelyn Mariuxi Bautista Guaranda

Cárdenas, R. V. (2004). Cohesión y adaptabilidad familiar y su relación con el rendimiento académico. Lima: Universidad Nacional Mayor de San Marcos.

Carmona Rodríguez, C., Sánchez Delgado, P., \& Bakieva, M. (2011). Actividades extraescolares y rendimiento académico: diferencias en auto concepto y género. Revista de Investigación Educativa, 447-465.

Cervini, R., Dari, N., \& Quiroz, S. (2014). Estructura familiar y rendimiento académico en países de américa latina. Investigación educativa, 569-597.

De Souza Barros, S., \& F. Elia, M. (2014). Las actitudes de los profesores: cómo influyen en la realidad de la clase. Universidad Federal de Río de Janeiro, 1-13.

Educación, M. d. (2012). Estándares de Calidad Educativa; Aprendizaje, Gestión, Desempeño Profesional e Infraestructura.

Educación, M. d. (2012). Estándares de Calidad Educativa; Aprendizaje, Gestión, Desempeño Profesional e Infraestructura.

Educacion, M. d. (2017). ¿Cómo se relaciona la infraestructura de la escuela con los aprendizajes de los estudiantes? Zoom educativo, 1-6.

Edwars Risopatron, V. (1991). EL CONCEPTO DE LA CALIDAD DE LA EDUCACION. Chile: UNESCO/ORELAC. Obtenido de http://unesdoc.unesco.org/images/0008/000884/088452SB.pdf

Erazo, O. A. (2012). El rendimiento académico, un fenómeno de múltiples relaciones y complejidades. Revista Vanguardia Psicológica, 144-173.

Fernández de la Reguera, J. A., \& Torres Martín, C. (2006). Una clave para la calidad de la institución educativa: Los planes de mejora. Educación y Educadores, 172-184.

Gonzales, B., Delma, M., Romero, H., Betsy, S., Serrano, Q., \& Estrella, C. (2014). "El material didáctico y su influencia en el aprendizaje significativo en los estudiantes del área ciencia, tecnologia y ambiente del cuarto grado de educación secundaria en el centro experimental de aplicación de la universidad nacional de educación. Lima.

Gregorat, Soria, García, \& Seco Maza. (2008). Rendimiento académico y nivel socioeconómico de los alumnos del ISEF de Catamarca.

Guerrero Serón, A. (2003). Las actividades extracolares y la innovación pedagógica como propiedades de la organización escolar y su incidencia en el aprendizaje escolar. Estudio de casos. Universidad Complutense de Madrid, 1-24.

Gutiérrez Capulín, R., Díaz Otero, K., \& Román Reyes, R. (2016). El concepto de familia en México: una revisión desde la mirada antropológica y demográfica. Ciencia Ergo Sum, 23(3). 


\section{Calidad educativa, entorno familiar y su relación con el rendimiento académico de la enseñanza secundaria en centros educativos de la provincia de Manabí durante año 2017 -2018}

Vol. 3, núm. 2., (2019)

María José Ortiz Zurita; Jazmín Jessenia Peñafiel León; Gabriela Natalia Torres Jara; Diana Valeria Delgado Campuzano; Evelyn Mariuxi Bautista Guaranda

Guzmán Arteaga, R., \& Pacheco Lora, M. (2014). Comunicación familiar y desempeño académico en estudiantes universitarios. Revista del Instituto de Estudios en Educación Universidad del Norte, 81-91.

Izzedin Bouquet, R., \& Pachajoa Londoño, A. (2009). Pautas, prácticas y creencias acerca de crianza... ayer y hoy. Liberabit, 1-8.

Kellaghan, T., Greaney, V., \& Murray, T. (2016). Utilización de los resultados de una evaluación nacional del rendimiento académico. Washington, D: Banco Internacional de Reconstrucción y Fomento/Banco Mundial.

Leal González, H. E. (1994). Factores sociofamiliares que influyen en el rendimiento escolar. Monterrey, Nuevo Leon: UANL.

León, A. (9 de agosto de octubre-diciembre de 2007). Qué es la educación. Educere, 11(39), 595-604.

López Pérez, N. (2015). La biblioteca escolar y su importancia dentro del aula de Educación Infantil. Facultad de Educación, 1-44.

López, A. A., González Barbera, C., \& Caso Niebla, J. (2016). Familia y rendimiento académico: su configuración de perfiles estudiantiles en secundaria. Revista Electrónica de Investigación Educativa, 18(1).

López, I. S. (2013). Apoyo parental y rendimiento académico. Victoria, Tamaulipas: Universidad Autónoma de Tamaulipas.

Merino, M., \& Morales, F. (2002). “Cuadernillos para la reflexión pedagógica: Participación de la familia”. Ministerio de Educación.

Molina, L., Pérez Pellín, S., Suárez, A., A, W., \& G, R. (2007). La importancia de formar en valores en la educacion superior. Universidad central de Venezuela, 1-8.

Molina, M. F., Messoulam, N., \& Schmidt, V. (2006). La familia como fuente de apoyo para un adecuado desempeño académico. XIII Jornadas de Investigación y Segundo Encuentro de Investigadores en Psicología del Mercosur. Buenos Aires: Facultad de Psicología, Universidad de Buenos Aires.

Navarro, R. E. (2003). El rendimiento académico: concepto, investigación y desarrollo. REICE. Revista Electrónica Iberoamericana sobre Calidad, Eficacia y Cambio en Educación, 1(2).

Ocaña Fernandez, Y. (2012). Influencia de los medios y materiales didácticos y el rendimiento académico de los alumnos de la asignatura filosofía y ética de la Universidad César Vallejo sedeLima Norteperiodo 2012-I. Lima.

Ortiz Zavaleta, M. d., \& Moreno Almazán, O. (2015). Estilos parentales: implicaciones sobre el rendimiento escolar en alumnos de educación media. 1-22. 


\section{Calidad educativa, entorno familiar y su relación con el rendimiento académico de la enseñanza secundaria en centros educativos de la provincia de Manabí durante año 2017 -2018}

Vol. 3, núm. 2., (2019)

María José Ortiz Zurita; Jazmín Jessenia Peñafiel León; Gabriela Natalia Torres Jara; Diana Valeria Delgado Campuzano; Evelyn Mariuxi Bautista Guaranda

Ortiz, Y. G., López de Castro Machado, D., \& Rivero Frutos, O. (2014). Estudiantes universitarios con bajo rendimiento económico ¿qué hacer? Edumecentro, 6(2).

Peceros, M., Fiorella, A., Reyes, I., \& Grecia, S. (2015). Familia monoparental y rendimiento academico. Peru.

Peréz Guitiérrez, A., \& Florido Bacallao, R. (2003). INTERNET: UN RECURSO EDUCATIVO. Etic@net, 1-12.

Ramírez, M. A. (2005). Padres y desarrollo de los hijos: prácticas de crianza. Estudios Pedagógicos XXXI, N 2: 167-177, 2005, 1-8.

Rico Alonzo, A. (1999). Formas, cambios y tendencias en la organización familiar en colombo. NOMADAS, 110-117.

Robledo Ramón, P., \& García Sánchez, J.-N. (2009). El entorno familiar y su influencia en el rendimiento académico de los alumnos con dificultades en el aprendizaje: revisión de estudios empíricos. Aula Abierta, 117-128.

Romagnoli, C., \& Cortese, I. (2016). Cómo la familia influye en el aprendizaje y rendimiento escolar. Volaras UC, 1-9.

Ruiz de Miguel, C. (2001). Factores familiares viinculados al bajo rendimiento. Revista Complutense de Eduación, 12(1), 81-113.

Sarramona, J. (1989). Fundamentos de la educación. España: CEAC.

Suárez, N., Tuero-Herrero, E., Bernardo, A., Fernández, E., Cerezo, R., González-Pienda, J. Núñez, J. (2011). El fracaso escolar en Educación Secundaria: Análisis del papel de la implicación familiar. Revista de Formación del Profesorado e Investigación Educativa, 49-64.

Teixidó, J. (1999). La comunicación en los Centros Educativos. 1-52.

Tejada, Á. (2006). Lectura y bibliotecas escolares en el Perú: Reflexiones y experiencias desde el sur del pais. Biblios, 1-19.

Tejedor Tejedor, F. J., \& Caride Gómez, J. A. (1988). Influencia de las variables contextuales en el rendimiento académico. Revista de Educación (287), 113-146.

Tilano, L. M., Henao, G. C., \& Restrepo, J. A. (2009). Prácticas educativas familiares y desempeño académico en adolescentes escolarizados en el grado noveno de instituciones educativas oficiales del municipio de Envigado. Revista de Ciencias Ssociales, 1-17.

Universidad de Nebrija. (2012). Estudio sobre la influencia de la iluminación en el rendimiento escolar. Luces CEI, 14-16. 


\section{Calidad educativa, entorno familiar y su relación con el rendimiento académico de la enseñanza secundaria en centros educativos de la provincia de Manabí durante año 2017 -2018}

Vol. 3, núm. 2., (2019)

María José Ortiz Zurita; Jazmín Jessenia Peñafiel León; Gabriela Natalia Torres Jara; Diana Valeria Delgado Campuzano; Evelyn Mariuxi Bautista Guaranda

Valdivia Sanchez, C. (2008). La familia: concepto, cambios y nuevos modelos. La Revue du REDIF, 1522.

Vasquez Tasayco, A. (2013). Calidad y calidad educativa. Investigación educativa, 49-71.

Vélez, E., Schiefelbein, E., \& Valenzuela, J. (1994). Factores que Afectan el Rendimiento Académico en la Educación Primaria. Revista latinoamericana de Innovaciones Educativas., 1-16.

Venegas Jiménez, P. (2004). Planificación educativa bases metodológicas para su desarrollo en el siglo $X X I$. Universidad Estatal a Distancia. 